\title{
Tumor Localization and Biodistribution with Radiolabeled Monoclonal Antibody against Pancreatic Cancer in Tumor-Bearing Nude Mice
}

\author{
Yong S. Chung, Tetsuji Sawada, Yasuyuki Kondo, \\ Jenny J.L. Ho*, Young S. Kim* and Michio Sowa \\ The First Department of Surgery, Osaka City University \\ Medical School and *Gastrointestinal Research Laboratory, \\ Veteran Administration Medical Center, University of \\ California, San Francisco, USA
}

\begin{abstract}
Chung, Y.S., Sawada, T., Kondo, Y., Ho, J.J.L., Kim, Y.S. and Sowa, M. Tumor Localization and Biodistribution with Radiolabeled Monoclonal Antibody against Pancreatic Cancer in Tumor-Bearing Nude Mice. Tohoku J. Exp. Med., 1992, $168(2), 397-401-\mathrm{Nd} 2$ is a murine monoclonal antibody produced against a mucin fraction purified from xenografts of a human pancreatic cancer cell line SW1990. Immunoperoxidase staining showed that the antigen recognized by Nd2 was psesent in $82.9 \%$ of pancreatic cancer tissues but not in tissues of normal pancreas and chronic pancreatitis. However $\mathrm{Nd} 2$ antigen was found not to be elevated in the sera of patients with pancreatic cancer. Four days after injection of ${ }^{111} \mathrm{In}-\mathrm{Nd} 2$ into athymic nude mice bearing SW1990 xenograft there was a higher accumulation in the tumor compared to ${ }^{111} \mathrm{In}$-normal mouse IgG1. When these mice were scanned with a gamma camera, labeled $\mathrm{Nd} 2$ was shown to accumulate in the tumor rapidly on the 1st day after injection and by the 4th day tumor accumulation was more distinctly visualized than non-specific accumulation in liver. These results indicate that Nd2 has high specificity and reactivity for pancreatic cancer and may have possible applicants in radioimmunodetection or targeting of therpeutic drugs in pancreatic cancer. $\mathrm{Nd} 2$; murine monoclonal antibody; immunoperoxidase reaction human pancreatic cancer cells SW 1990 ; radioimmunodetection
\end{abstract}

While the serological detection of pancreatic cancer show promise (Chung et al. 1987), another immunological approach to detection is radioimaging the tumor. Successful reports of MoAbs in radioimmunodetection have increased steadily for a number of malignancies. However, only a few MoAbs have been described for pancreatic cancer. $\mathrm{Nd} 2$ is a murine MoAb produced against mucins purified from xenografts of a human pancreatic cancer cell line SW1990. Nd2 has been reported to have specificity for pancreatic cancer and its reactivity appeared to be dependent on the integrity of the mucin protein core (Ho et al. 1991). In the

Address for reprints : 1-5-7 Asahi-machi, Abeno-ku, Osaka 545, Japan. 
present study we describe the specificity for pancreatic cancer of $\mathrm{Nd} 2$ and the biodistribution of ${ }^{111} \mathrm{In}$-labeled $\mathrm{Nd} 2$ in athymic nude mice bearing human pancreatic cancer xenografts.

\section{Matertals and Methods}

Antibody and radiolabeling. Nd2 hybridomas were injected I.P. into Balb/C mice and $\mathrm{Nd} 2$ was purified from ascites of mice by affinity chromatography on protein A columns. Normal murine immunoglobulin (IgG1) was used as a nonspecific control antibody. The chloramine $\mathrm{T}$ method was employed for the radioiodination of $\mathrm{Nd} 2$ with ${ }^{125} \mathrm{I}$. Radioactivity of ${ }^{125} \mathrm{I}-\mathrm{Nd} 2$ was $1 \mathrm{mCi} / \mathrm{mg}$. DTPA-conjugated $\mathrm{Nd} 2$ was aliquoted before ${ }^{111} \mathrm{In}$-labeling and ${ }^{111} \mathrm{InCl}_{3}$ were added when using. The labeling efficiency was more than $90 \%$ and specific activity of ${ }^{111} \mathrm{In}-\mathrm{Nd} 2$ and control mouse IgG1 was $1 \mathrm{mCi} / \mathrm{mg}$.

Immunohistochemistry. Immunoperoxidase staining of tissues was performed by the avidin-biotin-peroxidase complex method.

Radioimmunoassay. Sandwich radioimmunoassay were used for the screening of Nd2 antigen. SW1990 mucin, culture medium of pancreatic cancer cells (SW1990, CAPAN-2, RWP-1, RWP-2 and OCUP-1), serum from patients with pancreatic cancer and normal human serum were tested.

Biodistribution and immunoimaging. ${ }^{111} \mathrm{In}-\mathrm{Nd} 2$ and ${ }^{111} \mathrm{In}-\mathrm{normal}$ mouse IgG1 as a control were injected into nude mice bearing SW 1990 xenografts. Mice were sacrificed 4 days after injection and tumor, liver, blood, spleen and other tissues were weighed and radioactivity were determined. The biodistribution data was expressed as a percentage of injected dose per gram of tissue $(\% \mathrm{ID} / \mathrm{g})$. Immunoscintigrams were obtained with a gamma-camera equipped with a pinhole collimator under anesthesia.

\section{Results}

Immunohistochemical reactivity. $\mathrm{Nd} 2$ reactivity was observed with high percentage of pancreatic cancer specimens. Well and moderately differentiated cancer had much higher Nd2 reactivity compared to poorly differentiated cancer. However, Nd2 reactivity could not be detected with the specimens of normal pancreas and chronic pancreatitis except for a weak staining of the apical membrane of a few normal duct (Table 1).

TABLE 1. Immunoreactivity of Nd2

\begin{tabular}{ccc}
\hline Object & \multicolumn{2}{c}{ Positive ratio } \\
\hline Pancreatic cancer & $29 / 35$ & $82.9 \%$ \\
Differentiation & & \\
Well & $13 / 14$ & $93 \%$ \\
Moderate & $11 / 12$ & $92 \%$ \\
Poor & $5 / 9$ & $56 \%$ \\
Xenograft & $4 / 6$ & $66.7 \%$ \\
Chronic pancreatitis & $0 / 13$ & $0 \%$ \\
Normal pancreas & $0 / 21$ & $0 \%$ \\
\hline
\end{tabular}


Radiolabeled Monoclonal Antibody against Pancreatic Cancer

TABLE 2. Sandwich RIA of Nd2 antigen

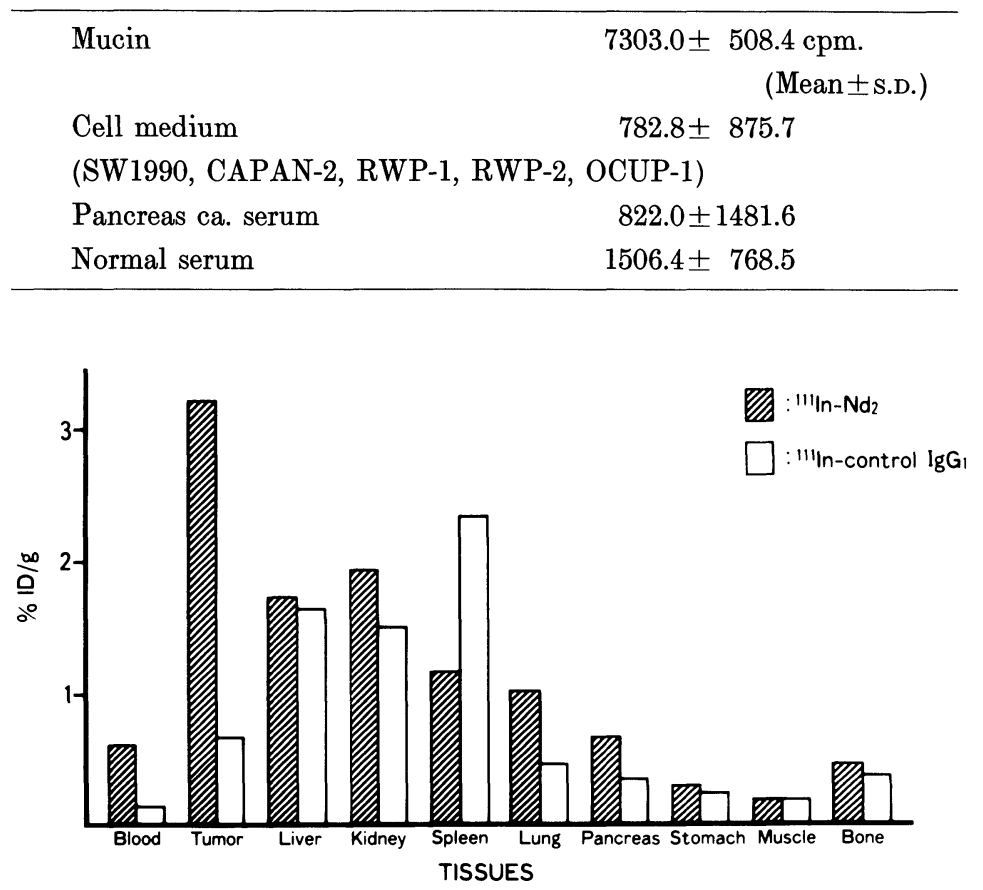

Fig. 1. Biodistribution $(\% \mathrm{ID} / \mathrm{g})$ of ${ }^{111} \mathrm{In}$-labeled $\mathrm{Nd} 2$ and ${ }^{111} \mathrm{In}$-labeled control mouse IgG1 in nude mice bearing SW1990 tumor, 4 days after injection.

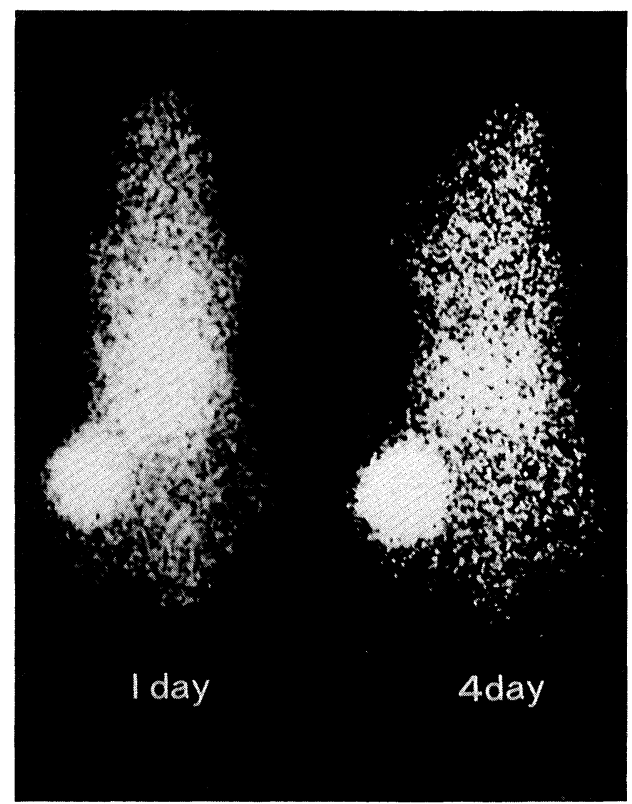

Fig. 2. Scintigrams of nude mice bearing SW1990 tumor, 1, 4 days after injection of ${ }^{111} \mathrm{In}$-labeled Nd2. 
Nd2 antigen in sera. Nd2 RIA levels in serum of pancreatic cancer patients was not different from that in normal serum. Culture medium of several kinds of pancreatic cancer cell lines also had similar low levels. However, Nd2 reactivity with SW1990 mucins was high (Table 2).

Biodistribution and immunoimaging. When expressed as $\%$ ID $/ \mathrm{g}$, the distribution of ${ }^{111} \mathrm{In}-\mathrm{Nd} 2$ (50 $\mu \mathrm{Ci} /$ mouse) in tumor was $3.18+1.86 \% \mathrm{ID} / \mathrm{g}$, five times as much as the control IgG1. In the other tissues, however, ${ }^{11} \mathrm{In}-\mathrm{Nd} 2$ and control IgG1 had similar values, liver (1.72 and 1.58), kidney (1.91 and 1.44) and spleen (1.15 and 2.28) (Fig. 1).

When a nude mouse bearing SW1990 xenograft was scanned 1 day after administration of ${ }^{111} \mathrm{In}-\mathrm{Nd} 2(50 \mu \mathrm{Ci})$, tumor imaging could be recognized despite a high background and liver accumulation. On the 4th day after injection, the background had decreased but labeled Nd2 remained high in the tumor, and tumor imaging was detected more distinctly than non-specific accumulation in liver. On the other hand, ${ }^{11}$ In-control IgG1 accumulated only in the liver and tumor imaging was not observed (Fig. 2).

\section{Discussion}

In this study $\mathrm{Nd} 2$ showed high specificity and reactivity for pancreatic cancer immunohistochemically. Furthermore, Nd2 antigen was not detected in sera of patients with pancreatic cancer and in culture medium of pancreatic cancer cell line suggest that $\mathrm{Nd} 2$ antigen does not have the characteristics of circulating antigen. Several studies reported that once the labeled MoAbs are injected intravascularly, antigen-antibody complexes are formed and removed by the reticuloendothelial system, which may result in decreased tumor uptake and increased liver uptake. Production of human anti mouse antibodies (HAMA) are also problem. MoAbs which recognize antigens that do not circulate in sera have the advantage because it is not necessary to inject unlabeled along with labeled MoAbs for immunoimaging. The present study suggests that $\mathrm{Nd} 2$ can be used in low doses. The lower dosages may reduce the likelihood of HAMA production. The tumor uptake ( $\% \mathrm{ID} / \mathrm{g})$ of ${ }^{111} \mathrm{In}-\mathrm{Nd} 2$ was not high compared to other reports, however in immunoimaging, good contrast between the image of the tumor and of the other organs is what is important and we have shown that Nd2 has the specificity required. For therapeutic applications greater accumulation of $\mathrm{Nd} 2$ might be achieved by use of antibody fragments, for example. In conclusion, the results of this study show that MoAb Nd2 has specificity for pancreatic cancer both in screening of fixed tissues as well as in vitro and in nude mice. Thus Nd2 may have clinical potential in immunodetection and immunotherapy in pancreatic cancer.

\section{Acknowledgments}

This work was supported in part by Grants-in-Aid from the Ministry of Education, 
Science and Culture of Japan.

\section{References}

1) Chung, Y.S., Ho, J.J.L., Kim, Y.S., Tanaka, H., Nakata, B., Hiura, A., Motoyoshi, H., Satake, K. \& Umeyama, K. (1987) The detection of human pancreatic cancerassociated antigen in the serum of cancer patients. Cancer, 60, 1636-1643.

2) Ho, J.J.L., Bi, N., Yan, P., Yuan, M., Norton, K.A. \& Kim, Y.S. (1991) Characterization of new pancreatic cancer-reactive monoclonal antibodies directed against purified mucin. Cancer Res., 51, 371-380. 